\title{
La Société suisse de dermatologie et de vénéréologie
}

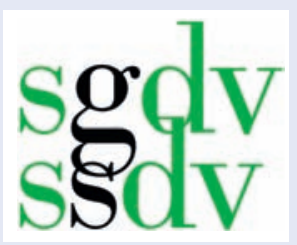

Pour la SSDV:

Prof. Peter Itin,

past-président, Bâle
Les maladies de la peau sont extraordinairement fréquentes et péjorent souvent gravement la qualité de vie. $20 \%$ de la population souffrent d'une affection cutanée nécessitant un traitement et 3,9\% des 80 millions de consultations médicales par année en Suisse sont occasionnés par des problèmes cutanés. $7 \%$ de toutes les ordonnances médicales concernent des médicaments dermatologiques.

La discipline de la dermatologie et de la vénéréologie comprend:

- l'anatomie, la physiologie, la pathologie macroscopique, dermatoscopique et microscopique de la peau et de ses annexes ainsi que des muqueuses du voisinage;

- les maladies vénériennes;

- les allergies cutanées, y compris le syndrome atopique;

- les tumeurs bénignes et malignes de la peau;

- les dermatoses dues au système vasculaire périphérique;

- les modifications cutanées dégénératives et celles dues à l'âge;

- la photobiologie, ainsi que toutes les procédures du diagnostic macroscopique, dermatoscopique, microscopique et microbiologique;

- toutes les thérapies scientifiquement reconnues, la prévention et la génétique des maladies cutanées.

Dans sa diversité, notre discipline compte plus de 2000 diagnostics différents et couvre aussi bien les affections somatiques que psychosomatiques de la peau. Elle s'occupe aussi des aspects psychosociaux et psychosexuels des dermatoses et des maladies vénériennes. Cette énumération montre que la dermatologie et la vénéréologie sont fortement interdisciplinaires et qu'il leur faut absolument collaborer avec toutes les disciplines médicales pour garantir une prise en charge thérapeutique optimale des patients.

Le dermatologue détermine les affections dermatologiques et sexuelles susmentionnées, prodigue les traitements et s'occupe de prévention.

La Société suisse de dermatologie et de vénéréologie (SSDV) a été fondée en 1913 dans le but de promouvoir la recherche scientifique et le traitement de la peau dans tous les domaines. La SSDV compte aujourd'hui 391 spécialistes en activité.
La SSDV défend les préoccupations et les intérêts de politique professionnelle de ses membres, de la société et de l'ensemble de la discipline. Un de ses objectifs principaux est d'assurer une formation postgraduée structurée, propre à transmettre le savoir et les aptitudes pratiques nécessaires, qu'elle évalue dans le cadre de l'examen de spécialiste. Elle veille également à la formation continue de ses membres et en organise le contrôle régulier. La SSDV est rattachée à des réseaux internationaux et promeut les échanges scientifiques dans le domaine de la dermatologie et de la vénéréologie.

La SSDV organise deux fois par année un congrès national en vue du transfert des connaissances mais aussi dans l'intention de permettre à ses membres de cultiver les contacts personnels. Elle publie deux revues: «Dermatologica Helvetica», qui est son organe officiel et «Dermatology», qui est une publication scientifique.

La formation postgraduée, complètement axée sur la discipline, dure cinq ans. Les candidats au titre de spécialiste peuvent faire valider une formation en angiologie et allergologie/immunologie clinique de six mois au maximum.

La SSDV poursuit les objectifs suivants:

1. promouvoir la dermatologie et de la vénéréologie dans les domaines cliniques et scientifiques;

2. encourager la relève;

3. assurer la qualité dans le domaine de la dermatologie et de la vénéréologie;

4. défendre les intérêts de politique professionnelle;

5. promouvoir la mise en réseau nationale et internationale;

6. garantir la fourniture de soins à la population.

Pour de plus amples informations:

Président:

Dr Thomas Hofer, Wettingen

Vice-président:

Dr Stephan Lautenschlager, Zurich

www.derma.ch 